\title{
An Analysis of Mode III Doubly Periodic Crack-Tip Field of Orthotropic Composite Materials
}

\author{
Wenbin Zhao, Chan Li, Xuexia Zhang, Hailing Xie, and Lujuan Yu \\ School of Applied Science, Taiyuan University of Science and Technology, Taiyuan 030024, China \\ Correspondence should be addressed to Xuexia Zhang; xuexiaz@126.com
}

Received 27 May 2013; Accepted 15 July 2013

Academic Editor: Ray Su

Copyright (C) 2013 Wenbin Zhao et al. This is an open access article distributed under the Creative Commons Attribution License, which permits unrestricted use, distribution, and reproduction in any medium, provided the original work is properly cited.

\begin{abstract}
The mechanical behavior near the doubly periodic crack tips for orthotropic composite materials plate subjected to antiplane shear loading is studied. This is done by complex function theory and conformal mapping of the Jacobi elliptic function with the help of boundary conditions. The analytical solution of the crack-tips stress intensity factor and the expression of stress fields are obtained. Numerical examples are given to analyze the impact of the different transverse spacing, longitudinal spacing, and the ratio of cracks periods on stress intensity factors. The results show that the crack-tip field increases with reducing either the transverse spacing or the longitudinal spacing. At the same time, the crack-tip field increases with the decrease of the ratio of cracks periods. This shows that the distribution form makes an important effect on the crack-tip field, but the crack density parameter is not the only cause.
\end{abstract}

\section{Introduction}

Fiber reinforced composite materials are used widely and are characterized by the proportion of small, specific strength and large specific modulus, but there are usually many microcracks, microholes in the process of fatigue damage. Because of these microcracks, microholes are arranged in periodic characteristic more or less, and studying the interference between the doubly periodic cracks helps to deepen the understanding of the materials failure mechanism and plays an important role in fracture behavior of materials.

Doubly periodic crack problem is an important mechanical model for the study of the interaction between multiple cracks. The more important value of doubly periodic crack model is that it provides the limit properties of interference between multiple cracks in the process from disorder to order. This problem is solved by the superposition method, the single-cell method, and numerical methods. By using distribution dislocation method [1], the doubly periodic distribution of crack problem is turned into the integral equation. Pseudotraction method [2,3] is also a good method to deal with this problem, but one needs to pay attention to the summation order and convergence issues. Chen and Lee [4], Chen and Lin [5], Dong and Lee [6], Yan and Jiang [7],
Yan et al. [8], and Tong et al. [9] used the eigen function expansion variational method to solve the doubly periodic cracks and analyze the interaction of stress intensity factor in the different cracks arrangement of the corresponding rule. In 2011, Xiao et al. [10] obtained the exact solution to the antiplane problem of doubly periodic conducting rigid line inclusions of unequal size in piezoelectric materials. Chang et al. [11] discussed the anti-plane problem of doubly periodic cracks in the infinite homogeneous piezoelectric materials using conformal mapping and elliptic function and calculated the stress intensity factor and stress field in a closed form solution. Using two-dimensional boundary element method, Pasternak [12] analysed the intensity of mechanical, electric, and magnetic fields near the tips of magnetoelectroelastic medium containing doubly periodic sets of cracks or thin inclusions. Wang [13] and Wang and Feng [14] introduced a famous summation equation of complex variable function series with infinite number of terms and solved the interaction of multiple rows of periodic cracks with extremely high accuracy.

In this paper, the cracks-tip field of Mode III double periodic cracks on plate subjected to anti-plane shear stress is studied through introducing the Westergaard stress function and conformal mapping of the Jacobi elliptic function. Stress 
intensity factor and stress fields on the cracks tip are obtained with the help of the boundary conditions. Numerical examples analyzed the impact of the different transverse spacing, longitudinal spacing, and the ratio of cracks periods on stress intensity factors. This research contributes to the understanding of the interaction between multiple cracks contained in the composite materials.

\section{Crack Model}

Figure 1 shows the infinite orthotropic composite plate with doubly periodic cracks. The crack length is $2 a$, transverse spacing is $b$, longitudinal spacing is $h$, and the plate surface is subjected to the shear force $\tau$.

From the theory of elasticity, strain-stress relationship $[15-18]$ is

$$
\begin{array}{cc}
\tau_{y z}=Q_{44} \gamma_{y z}, & \tau_{z x}=Q_{55} \gamma_{z x}, \\
\gamma_{y z}=\frac{\partial w}{\partial y}, & \gamma_{z x}=\frac{\partial w}{\partial x},
\end{array}
$$

where $Q_{44}, Q_{55}$ are the stiffness coefficients in the elastic principal direction of the orthotropic materials.

The balancing equation of the orthotropic materials can be expressed as follows:

$$
\frac{\partial \tau_{z x}}{\partial x}+\frac{\partial \tau_{y z}}{\partial y}=0 .
$$

By substituting (1) into (2), the governing equation can be written as

$$
Q_{55} \frac{\partial^{2} w}{\partial x^{2}}+Q_{44} \frac{\partial^{2} w}{\partial y^{2}}=0 .
$$

Assuming $w=w(x+s y)$, the characteristic equation of the governing equation (3) can be obtained as follows:

$$
Q_{44} s^{2}+Q_{55}=0
$$

The roots of characteristic equation (4) are

$$
s_{1}=i \sqrt{\frac{Q_{55}}{Q_{44}}}=i \beta, \quad s_{2}=-i \beta .
$$

Labeling

$$
z_{1}=x+s_{1} y=x_{1}+i y_{1},
$$

where $x_{1}=x, y_{1}=\beta y$.

By (5) and (6), the governing equation (3) can be turned into generalized harmonic equation:

$$
\nabla_{1}^{2} w=\left(\frac{\partial^{2}}{\partial x_{1}^{2}}+\frac{\partial^{2}}{\partial y_{1}^{2}}\right) w=0
$$

By the complex function theory, the governing equation (3) has a real analytic solution. We can choose it as follows:

$$
w=\operatorname{Re}\left[F\left(z_{1}\right)\right] .
$$

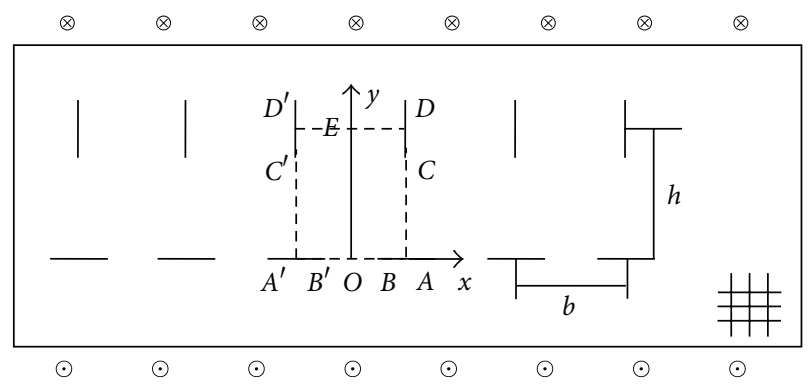

FIGURE 1: The crack model of doubly periodic cracks in the orthotropic composite plate.

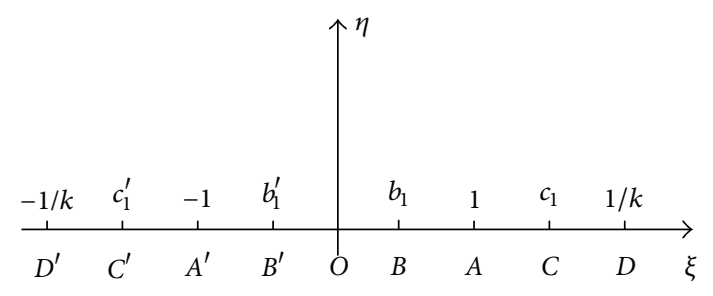

Figure 2: Conformal mapping.

Combining (1) with (8), the stress expression can be written as

$$
\tau_{x z}-i \tau_{y z}=Q_{55} \operatorname{Re}\left[F^{\prime}\left(z_{1}\right)\right]+i Q_{44} \beta \operatorname{Im}\left[F^{\prime}\left(z_{1}\right)\right] .
$$

Now, formulate the boundary conditions of the rectangle $O B A C D D^{\prime} C^{\prime} A^{\prime} B^{\prime}$. Here, $B A=C D=D^{\prime} C^{\prime}=A^{\prime} B^{\prime}=a$, $A^{\prime} A=b$, and $A D=h$. The crack faces are free of traction, and it follows that

$$
\begin{aligned}
& \tau_{y z}=0 \text { on the } B A, A^{\prime} B^{\prime}, \\
& \tau_{x z}=0 \text { on the } C D, D^{\prime} C^{\prime} .
\end{aligned}
$$

\section{The Conformal Mapping of Jacobi Elliptical Function}

Making use of the Jacobi elliptic function [19]

$$
\zeta=\operatorname{sn}\left(\frac{z_{1}}{A}, k\right)
$$

$z_{1}=A \int_{0}^{\zeta}\left(d t / \sqrt{\left(1-t^{2}\right)\left(1-k^{2} t^{2}\right)}\right)$ is an inverse function, where $A$ is a constant, and $k$ is the modulus of the function sn. The transform characteristic points in Figure 2 are indicated. With the relation of the elliptic function

$$
\frac{A^{\prime} A}{A D}=\frac{b}{h}=\frac{2 A K(k)}{A K\left(k^{\prime}\right)},
$$

where $k^{\prime 2}=1-k^{2}$ is called as complementary modulus, and $K(k)$ is the complete elliptic integral of the first kind:

$$
K(k)=\int_{0}^{1} \frac{d t}{\sqrt{\left(1-t^{2}\right)\left(1-k^{2} t^{2}\right)}} .
$$


From the mapping function, we can get that

$$
A=\frac{b}{2 K(k)}=\frac{h}{K\left(k^{\prime}\right)} \text {. }
$$

So,

$$
\frac{b}{h}=\frac{2 K(k)}{K\left(k^{\prime}\right)}=\frac{2 \int_{0}^{1}\left(d t / \sqrt{\left(1-t^{2}\right)\left(1-k^{2} t^{2}\right)}\right)}{\int_{0}^{1}\left(d t / \sqrt{\left(1-t^{2}\right)\left(1-k^{\prime 2} t^{2}\right)}\right)}
$$

The $k, k^{\prime}$ can be obtained by numerical calculation. Using formula (11), the parameters can be obtained as follows:

$$
\begin{aligned}
b_{1} & =\operatorname{sn}\left(\frac{(b / 2)-a}{A}, k\right) \\
& =\frac{\operatorname{cn}(a / A, k)}{\operatorname{dn}(a / A, k)} \\
& =-b_{1}^{\prime}, \\
c_{1} & =\operatorname{sn}\left(\frac{((b / 2)-a)+i(h-a)}{A}, k\right) \\
& =\frac{\operatorname{dn}\left(a / A, k^{\prime}\right)}{k} \\
& =-c_{1}^{\prime} .
\end{aligned}
$$

Here $\mathrm{cn}, \mathrm{dn}$ are Jacobi elliptic functions also.

\section{The Solution of Crack-Tip Stress Field}

Mapping the $z$-plane into $\zeta$-plane, the boundary conditions are

$$
\Phi(\zeta)=i F^{\prime}\left(z_{1}\right)
$$

On $\left(-1 / k, c_{1}^{\prime}\right),\left(-1, b_{1}^{\prime}\right),\left(b_{1}, 1\right)$, and $\left(c_{1}, 1 / k\right)$,

$$
Q_{44} \beta \operatorname{Re}[\Phi(\zeta)]=0 .
$$

On the remainder of the real axis,

$$
Q_{55} \operatorname{Im}[\Phi(\zeta)]=0
$$

Using the Keldych-Sedov formula [16], the solution of $\Phi(\zeta)$ is

$$
\Phi(\zeta)=c_{0} \sqrt{\frac{\left(\zeta^{2}-1\right)\left(\zeta^{2}-1 / k^{2}\right)}{\left(\zeta-c_{1}^{\prime}\right)\left(\zeta-b_{1}^{\prime}\right)\left(\zeta-c_{1}\right)\left(\zeta-b_{1}\right)}},
$$

where $c_{0}$ is an undetermined coefficient.

From (16), it can be simplified to

$$
\Phi(\zeta)=c_{0} \sqrt{\frac{\left(\zeta^{2}-1\right)\left(\zeta^{2}-1 / k^{2}\right)}{\left(\zeta-c_{1}^{2}\right)\left(\zeta-b_{1}^{2}\right)}} .
$$

Considering the periodic cracks on the $z$-plane, the balancing condition of shear force is as follows:

$$
\int_{B^{\prime}}^{B} \tau_{y z} d x=b \tau
$$

Substituting (11), (12), and (21) into (22),

$$
c_{0}=\frac{k K(k) \tau}{Q_{44} \beta K\left(k_{r}\right)}\left(c_{1}+b_{1}\right),
$$

where $k_{r}^{2}=4 c_{1} b_{1} /\left(c_{1}+b_{1}\right)^{2}$.

Substituting $c_{0}$ into (21) gets

$$
\Phi(\zeta)=\frac{k K(k) \tau}{Q_{44} \beta K\left(k_{r}\right)}\left(c_{1}+b_{1}\right) \sqrt{\frac{\left(\zeta^{2}-1\right)\left(\zeta^{2}-1 / k^{2}\right)}{\left(\zeta-c_{1}^{2}\right)\left(\zeta-b_{1}^{2}\right)}} .
$$

The stress field of double periodic cracks is

$$
\begin{aligned}
\tau_{y z} & =Q_{44} \beta \operatorname{Re}[\Phi(\zeta)] \\
& =\frac{k K(k) \tau}{K\left(k_{r}\right)}\left(c_{1}+b_{1}\right) \operatorname{Re}\left[\sqrt{\frac{\left(\zeta^{2}-1\right)\left(\zeta^{2}-1 / k^{2}\right)}{\left(\zeta-c_{1}^{2}\right)\left(\zeta-b_{1}^{2}\right)}}\right], \\
\tau_{x z} & =Q_{55} \operatorname{Im}[\Phi(\zeta)] \\
& =\frac{Q_{55} k K(k) \tau}{Q_{44} \beta K\left(k_{r}\right)}\left(c_{1}+b_{1}\right) \operatorname{Im}\left[\sqrt{\frac{\left(\zeta^{2}-1\right)\left(\zeta^{2}-1 / k^{2}\right)}{\left(\zeta-c_{1}^{2}\right)\left(\zeta-b_{1}^{2}\right)}}\right] .
\end{aligned}
$$

\section{Stress Intensity Factor and the Interaction between Cracks}

Considering periodicity, the stress intensity factor $K_{\mathrm{III} B}$ near the tip $B((b / 2)-a, 0)$ is defined by

$K_{\mathrm{III} B}$

$$
\begin{aligned}
& =\lim _{z \rightarrow(b / 2)-a} \sqrt{2 \pi\left(\frac{b}{2}-a-z\right) \tau_{y z}} \\
& =\frac{k^{\prime}}{K\left(k_{r}\right)} \sqrt{\frac{b K(k)\left(c_{1}+b_{1}\right)}{2 a b_{1}\left(c_{1}-b_{1}\right)}} \operatorname{Re}\left[\sqrt{\frac{\operatorname{sn}\left(a / A, k^{\prime}\right)}{\operatorname{dn}^{2}\left(a / A, k^{\prime}\right)}}\right] \sqrt{\pi a} \tau .
\end{aligned}
$$

Labeling

$$
K_{\mathrm{III} B}^{*}=\frac{k^{\prime}}{K\left(k_{r}\right)} \sqrt{\frac{b K(k)\left(c_{1}+b_{1}\right)}{2 a b_{1}\left(c_{1}-b_{1}\right)}} \operatorname{Re}\left[\sqrt{\frac{\operatorname{sn}\left(a / A, k^{\prime}\right)}{\operatorname{dn}^{2}\left(a / A, k^{\prime}\right)}}\right] .
$$


The stress intensity factor $K_{\mathrm{IIIC}}$ near the tip $C(b / 2, h-a)$ is defined by

$$
\begin{aligned}
& K_{\text {IIIC }} \\
& =\lim _{z \rightarrow(b / 2)+i(h-a)} \sqrt{2 \pi\left[z-\left(\frac{b}{2}+i(h-a)\right)\right] \tau_{y z}} \\
& =\frac{K(k)}{K\left(k_{r}\right)} \\
& \times \operatorname{Re}\left[\sqrt{\frac{h K\left(k^{\prime}\right)\left(c_{1}+b_{1}\right)}{a c_{1}\left(c_{1}-b_{1}\right)}}\right. \\
& \left.\times \sqrt{\sqrt{1-\frac{\mathrm{dn}^{2}\left(a / A, k^{\prime}\right)}{k^{2}}} \sqrt{1-\operatorname{dn}^{2}\left(\frac{a}{A}, k^{\prime}\right)}}\right] \\
& \times \sqrt{\pi a} \tau . \\
& K_{\text {IIIC }}^{*} \\
& =\frac{K(k)}{K\left(k_{r}\right)} \\
& \times \operatorname{Re}\left[\sqrt{\frac{h K\left(k^{\prime}\right)\left(c_{1}+b_{1}\right)}{a c_{1}\left(c_{1}-b_{1}\right)}}\right. \\
& \times \sqrt{\sqrt{1-\frac{\mathrm{dn}^{2}\left(a / A, k^{\prime}\right)}{k^{2}}} \sqrt{1-\mathrm{dn}^{2}\left(\frac{a}{A}, k^{\prime}\right)}} .
\end{aligned}
$$

If the number of collinear cracks (density) increases, the strength of the crack tip field increases; similarly, if the number of parallel cracks (density) increases, the strength of the crack tip field increases. On the contrary, the crack tip field decreases with the increase of the period ratio $b / h$. It shows that the distribution form of cracks has an important effect on crack-tip field. For the cracks of rectangular distribution, under the condition of the different ratios $b / h$, the variation of the stress intensity factor of crack-tip $B$ is showed in Figures 3 and 4 . In Figure $3, K_{\mathrm{III} B}^{*}$ increases with increasing the ratio $2 a / b$. When $2 a / b \rightarrow 1, K_{\mathrm{III} B}^{*}$ grows quickly and tends to infinity. The reason is that the interaction is much smaller when the transverse spacing $b$ is longer in the initial phase of curve. In Figure $4, K_{\mathrm{III} B}^{*}$ turns large rapidly and tends to infinity with increasing the ratio $a / h$ under the condition of the different ratio $b / h$. At the end stage of curve, the longitudinal spacing is smaller, and the interaction is obvious. The cause is that the longitudinal spacing $h$ is reduced at the end stage of curve.

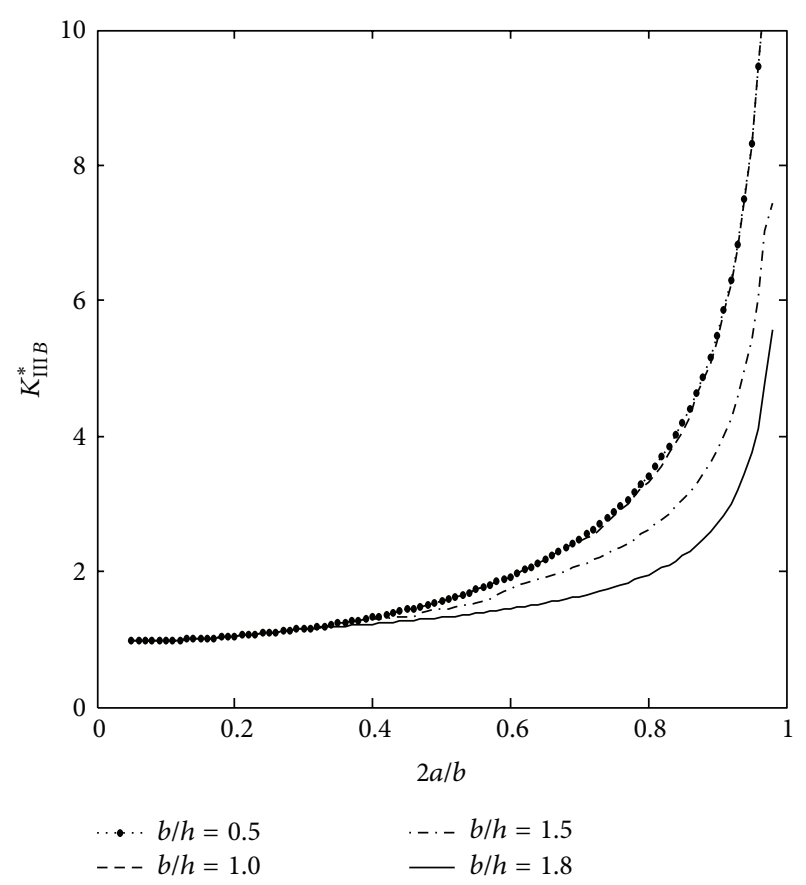

FIGURE 3: Various SIF of crack-tip $B$ with $2 a / b$.

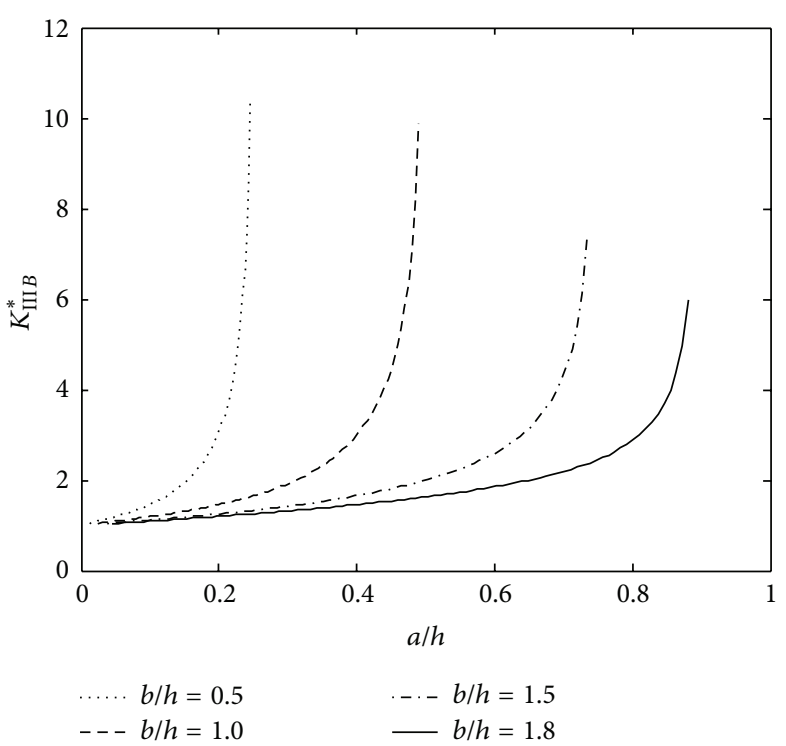

FIGURE 4: Various SIF of crack-tip $B$ with $a / h$ for $b / h=0.5,1.0,1.5$, and 1.8 .

The stress intensity factor of crack-tip $C$ is showed in Figure 5 . The variation of the $K_{\text {IIIC }}^{*}$ with the ratio $a / h$ increase is plotted in Figure 5 for several values of the dimensionless ratio $b / h$. With the ratio $b / h$ reduction, the $K_{\text {IIIC }}^{*}$ is increased; that is, the crack-tip $B$ makes an amplification effect on cracktip C. It can be seen from Figure 6 that the stress intensity factor in crack-tip $B$ has a strong interaction, which is caused by the distribution of crack-tip $C$ when the longitudinal spacing $h$ is smaller. However, when the longitudinal spacing 


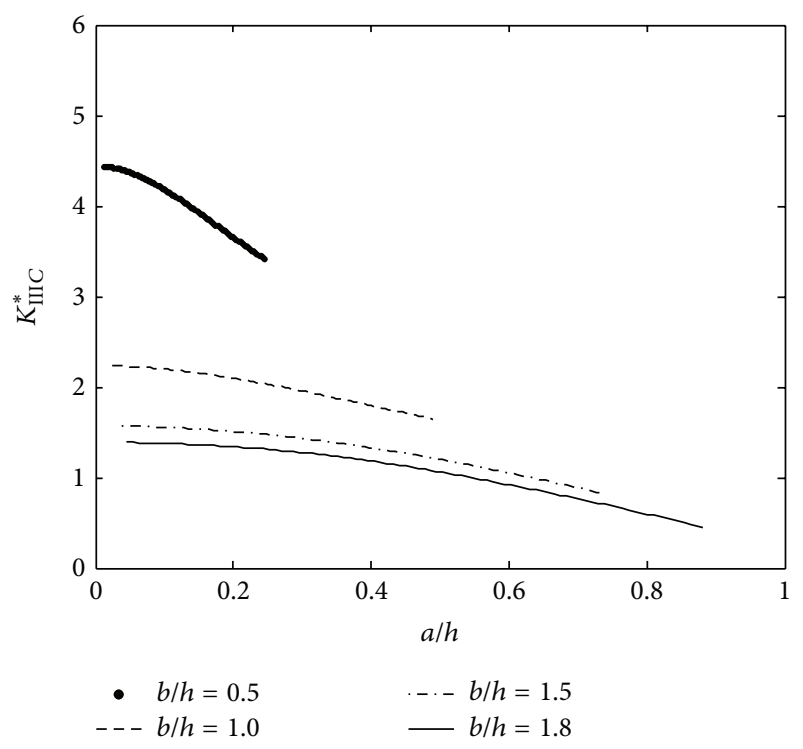

FIgURE 5: Various SIF of crack-tip $C$ with $a / h$.

$h$ is larger, the crack-tip field remains unchanged; that is, the stress intensity factor is unchanged within a certain range of the longitudinal spacing $h$. But, when $h$ grows bigger, the transverse spacing $b$ also increases, and the crack-tip field deduces; that is, the crack-tip $C$ field has a shielding effect on crack-tip $B$. Then, when $h$ grows relatively large, the changes in the crack-tip field are stabilized, which means that the crack tip $C$ has no interference effect on crack-tip $B$. From Figures 5 and 6 , we can deduce that the important influence of the crack distribution form on crack-tip field is not just caused by the crack density parameter.

\section{Conclusions}

(1) The analytical solution of the stress intensity factor of doubly periodic cracks in orthotropic composite materials is obtained under anti-plane mechanical load, by the introduction of the Jacobi elliptic function and the conformal mapping method.

(2) It is easy to calculate the analytic form of stress intensity factor through numerical calculation to obtain the modulus $k$, which contributes to a better understanding of the nature of the fault of the interaction between multiple cracks in composite materials.

(3) Analyses of the influence on stress intensity factor at different transverse spacing, longitudinal spacing, and the ratio $b / h$ are given. The results show that the important influence of the crack distribution form on crack-tip field is not just caused by the crack density parameter.

\section{Acknowledgments}

This work was financially supported by the Science and Technology Major Project of the Ministry of Education of China

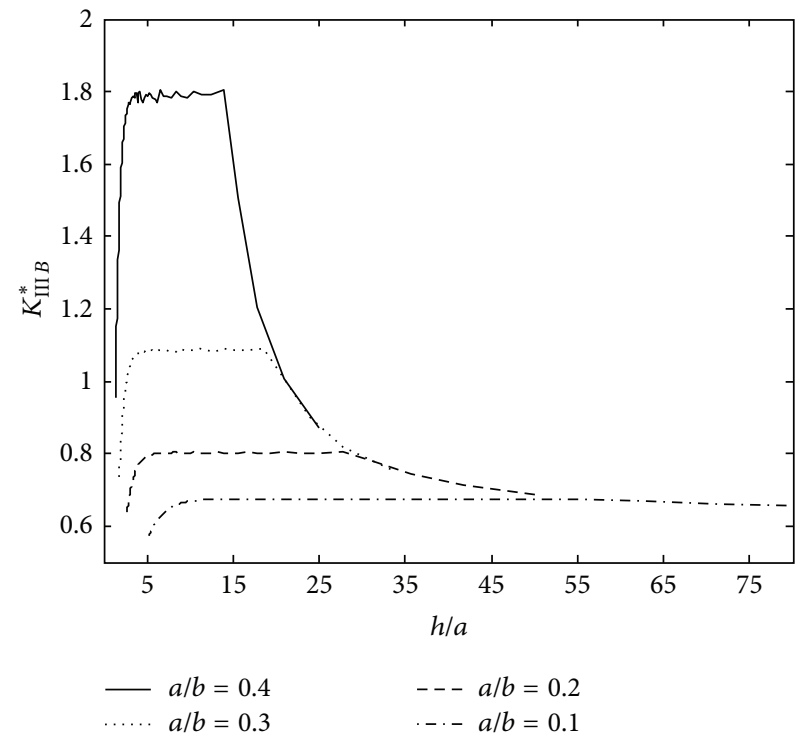

FIGURE 6: Various SIF of crack-tip $B$ with $h / a$ for $a / b=0.1,0.2,0.3$, and 0.4 .

(208022) and the Doctoral Initiating Project of the Taiyuan University of Science and Technology (20122005).

\section{References}

[1] W. R. Delameter, G. Herrman, and D. M. Barnett, "Weakening of an elastic solid by a rectangular array of cracks," Journal of Applied Mechanics, vol. 42, no. 1, pp. 74-80, 1975.

[2] G. S. Wang, "The interaction of doubly periodic cracks," Theoretical and Applied Fracture Mechanics, vol. 42, no. 3, pp. 249294, 2004.

[3] M. Isida and H. Igawa, "Doubly-periodic array and zig-zag array of cracks in solids under uniaxial tension," International Journal of Fracture, vol. 53, no. 3, pp. 249-260, 1992.

[4] Y. Z. Chen and K. Y. Lee, "An infinite plate weakened by periodic cracks," Journal of Applied Mechanics, vol. 69, no. 4, pp. 552-555, 2002.

[5] Y. Z. Chen and X. Y. Lin, "Eigenfunction expansion variational method for stress intensity factor and T-stress evaluation of a circular cracked plate," Acta Mechanica, vol. 196, no. 1-2, pp. 5573, 2008.

[6] C. Y. Dong and K. Y. Lee, "Numerical analysis of doubly periodic array of cracks/rigid-line inclusions in an infinite isotropic medium using the boundary integral equation method," International Journal of Fracture, vol. 133, no. 4, pp. 389-405, 2005.

[7] P. Yan and C. P. Jiang, "An eigenfunction expansion-variational method based on a unit cell in analysis of a generally doubly periodic array of cracks," Acta Mechanica, vol. 210, no. 1-2, pp. 117-134, 2010.

[8] P. Yan, F. L. Chen, C. P. Jiang, and F. Song, "An eigenfunction expansion-variational method in prediction of the transverse thermal conductivity of fiber reinforced composites considering interfacial characteristics," Composites Science and Technology, vol. 70, no. 12, pp. 1726-1732, 2010.

[9] Z. H. Tong, C. P. Jiang, S. H. Lo, and Y. K. Cheung, "A closed form solution to the antiplane problem of doubly periodic 
cracks of unequal size in piezoelectric materials," Mechanics of Materials, vol. 38, no. 4, pp. 269-286, 2006.

[10] J. H. Xiao, Y. L. Xu, and C. P. Jiang, "Exact solution to the antiplane problem of doubly periodic conducting rigid line inclusions of unequal size in piezoelectric materials," ZAMM Zeitschrift fur Angewandte Mathematik und Mechanik, vol. 91, no. 5, pp. 413-424, 2011.

[11] L. H. Chang, S. H. Ding, and X. Li J, "Anti-plane problems of doubly periodic cracks in piezoelectric materials," Journal of Ningxia University, vol. 32, no. 1, pp. 18-21, 2011.

[12] I. Pasternak, "Doubly periodic arrays of cracks and thin inhomogeneities in an infinite magnetoelectroelastic medium," Engineering Analysis with Boundary Elements, vol. 36, no. 5, pp. 799-811, 2012.

[13] G. S. Wang, "The interaction of doubly periodic cracks," Theoretical and Applied Fracture Mechanics, vol. 42, no. 3, pp. 249294, 2004.

[14] G. S. Wang and X. T. Feng, "The interaction of multiple rows of periodical cracks," International Journal of Fracture, vol. 110, no. 1, pp. 73-100, 2001.

[15] W. Y. Yang, J. L. Li, and X. X. Zhang, Complex Function Methods in Composite Materials Fracture, Science Press, Beijing, China, 2005.

[16] X. X. Zhang, X. C. Cui, W. Y. Yang, J. Li, and W. B. Zhao, "An analytical solution for interface cracks under the mode II condition," Advanced Science Letters, vol. 4, no. 3, pp. 1093-1097, 2011.

[17] X. X. Zhang, X. C. Cui, W. Y. Yang, and J. Li, "Crack-tip field on mode II interface crack of double dissimilar orthotropic composite materials," Applied Mathematics and Mechanics, vol. 30, no. 12, pp. 1489-1504, 2009.

[18] X. X. Zhang, C. Li, X. C. Cui, and W. B. Zhao, "Analysis of mode III collinear periodic cracks-tip stress field of an infinite orthotropic plate," Advanced Materials Research, vol. 446-449, pp. 2080-2084, 2012.

[19] Z. X. Wang and D. R. Guo, Introduction to Special Function, Science Press, Beijing, China, 1979. 


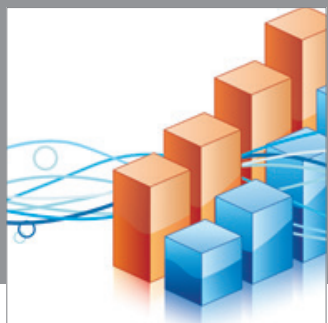

Advances in

Operations Research

mansans

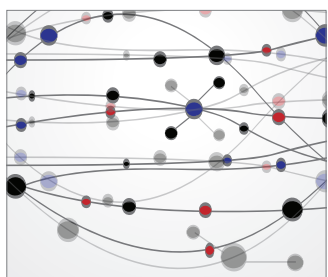

The Scientific World Journal
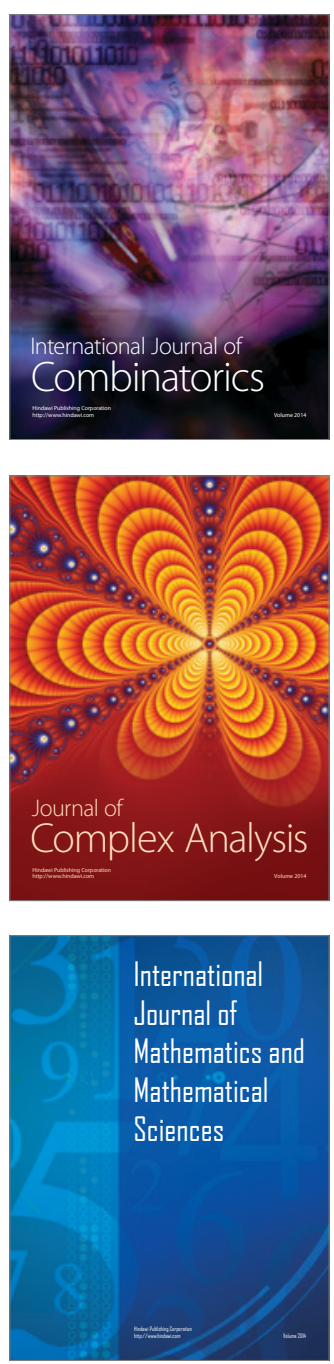
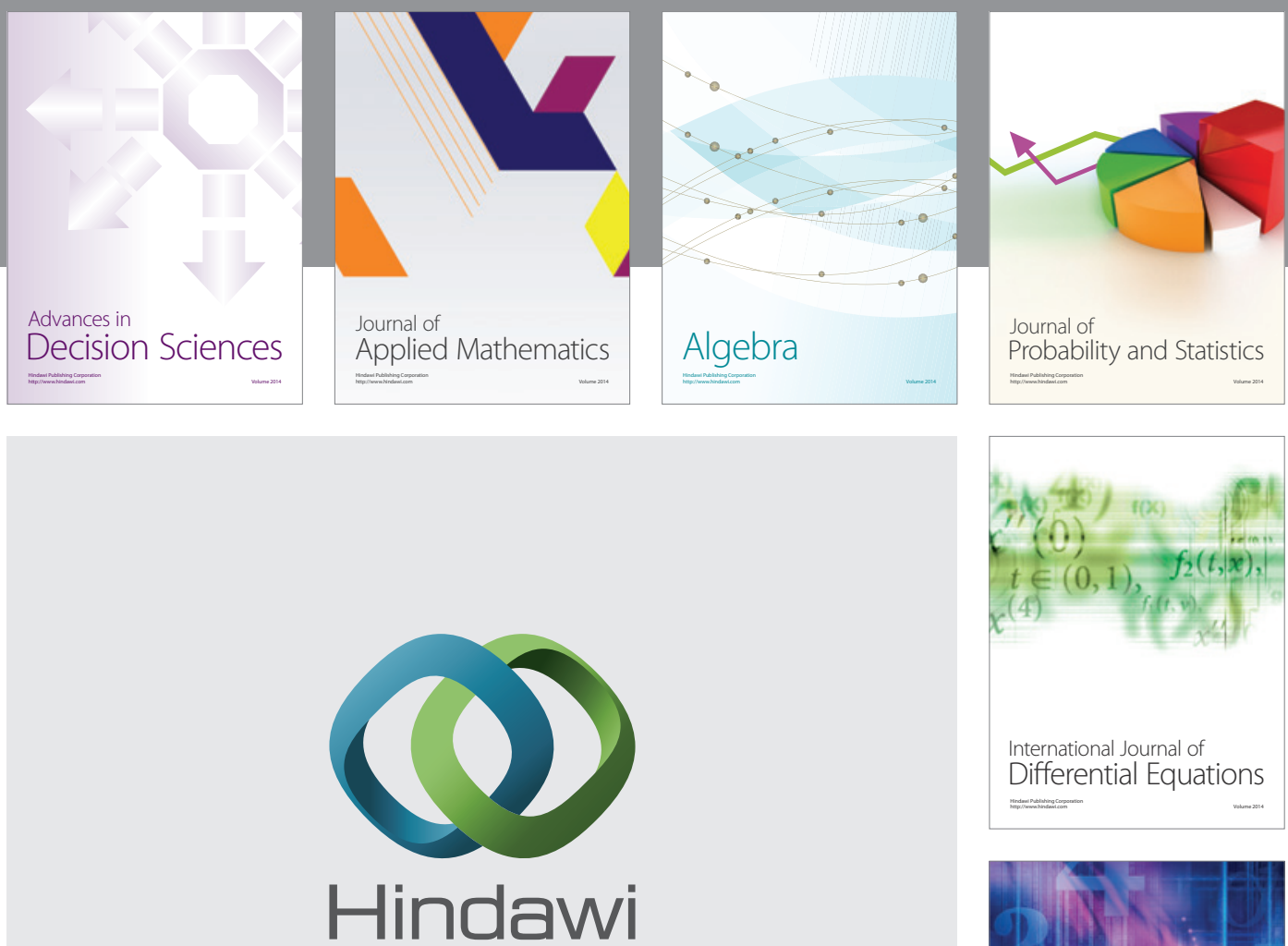

Submit your manuscripts at http://www.hindawi.com
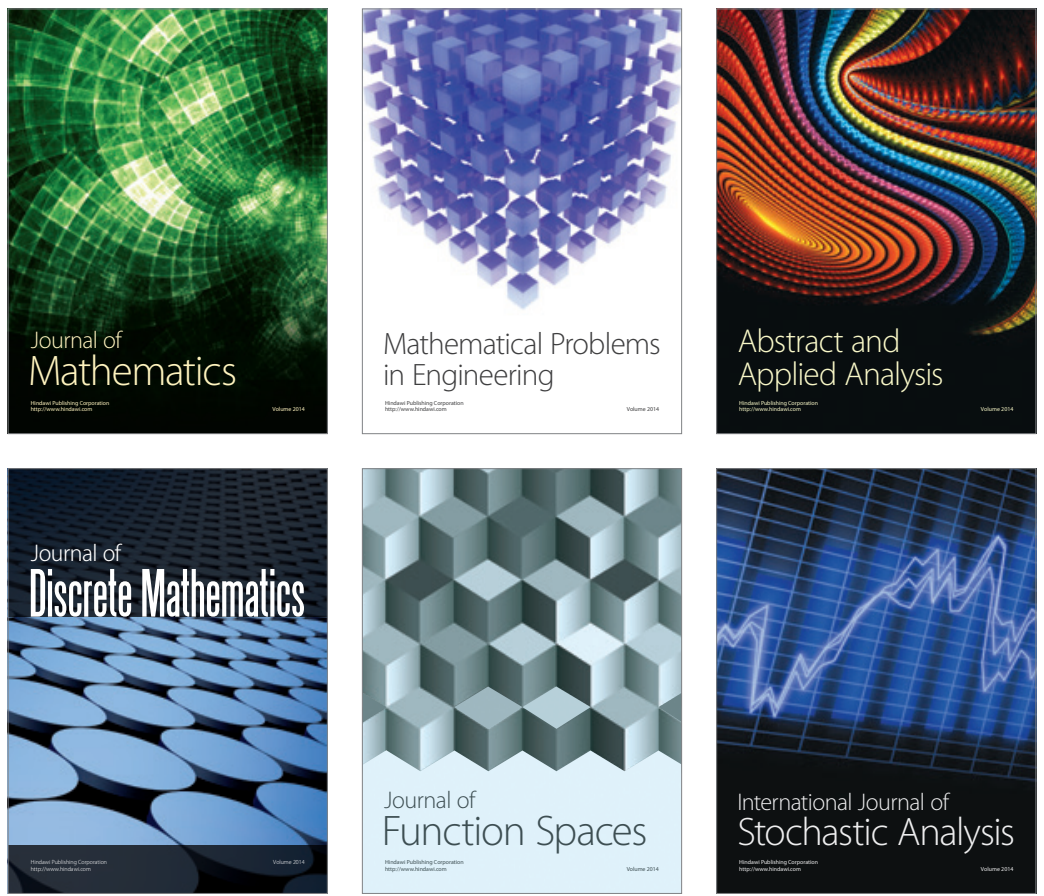

Journal of

Function Spaces

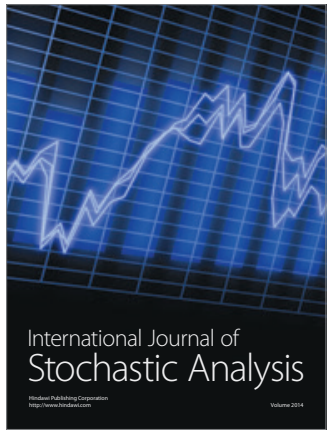

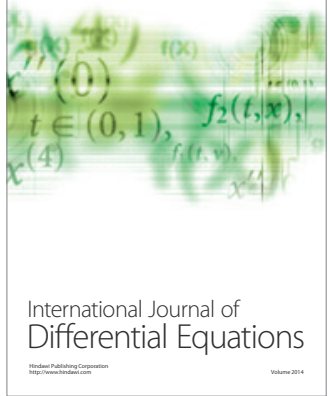
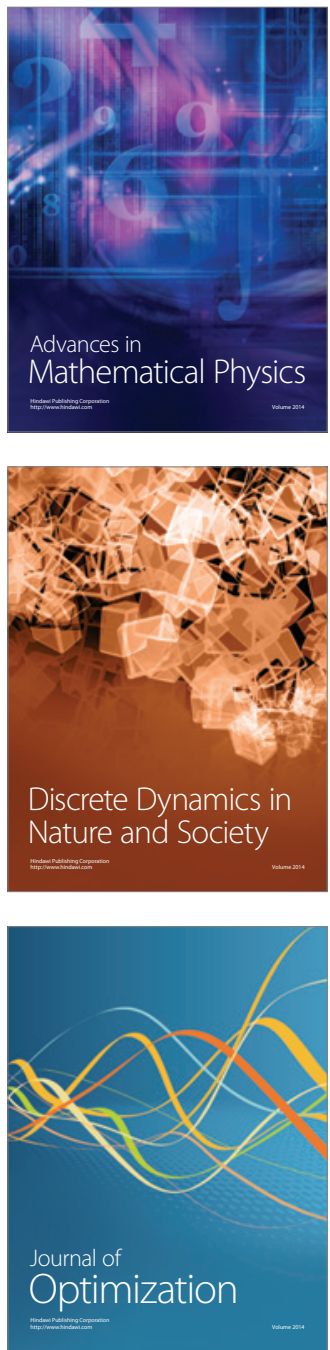